\title{
7. Laboratory Determination of the Luminous Efficiency of Meteor Constituents
}

\author{
Howard F. Savage and Charles A. Boitnott \\ Ames Research Center, NASA \\ Moffett Field, California
}

\begin{abstract}
$A$ crossed beam apparatus has been used to measure the emission and ionization cross sections for the prominent spectral features of $\mathrm{Na}, \mathrm{Ca}, \mathrm{Mg}$, and $\mathrm{Fe}$ in collisions with $\mathrm{N}_{2}$ and $\mathrm{O}_{2}$ over the velocity range of 30 to $120 \mathrm{~km} / \mathrm{s}$. From the emission and ionization cross sections, the absolute luminous efficiencies in air were determined over the range of meteor velocities. The maximum luminous efficiencies for the brightest features were: greater than 1 percent for the $\mathrm{Na} \mathrm{D}$-lines, 0.2 percent for the $\mathrm{CaI}(2)$ singlet, 0.06 percent for the $M g I(2)$ and $M g I(3)$ triplets, and 0.4 percent for $F e$ over the visible spectral range. These luminous efficiencies are valid for free molecular flow conditions for velocities above about $30 \mathrm{~km} / \mathrm{s}$ and are directly applicable to spectroscopic observations of faint meteors. The luminous efficiency of a typical stone will be presented and compared with the efficiencies determined by other investigators. In contrast to previous work, the luminous efficiency found for stone in the present investigation decreases with velocity above about $50 \mathrm{~km} / \mathrm{s}$.
\end{abstract}

$\mathbf{M}^{2}$ ETEOR LUMINOSITY RESULTS primarily from the excitation of vaporized meteoric constituents during decelerating collisions occurring with atmospheric molecules. The naturally occurring process of meteor vapor excitation during the cascade of energy-transferring collisions can be simulated in the laboratory by observing the emission probability under controlled single collision conditions and integrating over all collisions to obtain the total radiating efficiency.

The major aim of our research at Ames Research Center is to make appropriate laboratory measurements of the light emissions due to collisions between air molecules and typical meteoric atoms to allow for a direct calculation of the spectral luminosity of meteors. The efficiency of converting kinetic energy to luminous energy is the luminous efficiency, $\tau$, and for a particular spectral feature the two are related by

$$
I_{\lambda}=-1 / 2 \tau_{\lambda} v^{2} \frac{d m}{d t}
$$

where $v$ is the meteor velocity, $d m / d t$ is the mass loss rate of the element and $\lambda$ is the wavelength of the spectral feature.

The spectral luminous efficiency for free molecular flow conditions can be determined from the relationship given by Sida (1969)

$$
\tau_{\lambda}=\frac{2 E_{\lambda}}{E_{0}} \int_{E_{t}}^{E_{0}} \frac{\sigma_{\lambda}}{\sigma_{m}} \frac{d E}{E}
$$

where $E_{0}$ is the initial kinetic energy, $E_{\lambda}$ is the photon energy, $E_{t}$ is the threshold energy for exciting the wavelength $\lambda, \sigma_{m}$ is the momentum transfer cross section, and $\sigma_{\lambda}$ is the cross-section for the emission of a photon of wavelength $\lambda$ in a collision between an air molecule and a meteor atom. The mathematical formulation of $\tau$ in slip 
flow is too complex for present analysis and will not be considered here.

An appreciable fraction of the ablated meteor atoms is expected to be ionized during the deceleration process. The ions formed can produce either ion lines or, if neutralization occurs, neutral lines upon subsequent collisions. Therefore, an accurate determination of $\tau_{\lambda}$ must take into account the fraction of ions present during the deceleration process. The luminous efficiency equation can be evaluated using an effective emission cross section given by:

$$
\sigma_{\lambda}(E)=\sigma_{\lambda i}(E) \frac{N_{i}(E)}{N_{0}}+\sigma_{\lambda n}(E)\left[1-\frac{N_{i}(E)}{N_{0}}\right]
$$

where $\sigma_{\lambda i}(E)$ is the emission cross section for collisions of ions with air molecules and $\sigma_{\lambda n}(E)$ is the emission cross section for those collisions involving neutral metal atoms. The ion concentration generated in decelerating the ablated atoms from initial energy $E_{0}$ to energy $E$ can be found from the relation $N_{i}(E) / N_{0}=1-e^{-\beta(E)}$ where the ionization probability $\beta(E)$ is determined from the relation given by Sida (1969)

$$
\beta(E)=2 \int_{E}^{E_{0}} \frac{\sigma_{i}}{\sigma_{m}} \frac{d E}{E}
$$

where $\sigma_{i}$ is the ionization cross section. Finally, the measurable quantities in our experiments, which are related to $\sigma_{\lambda}$ and $\sigma_{i}$, are the number of photons and ions produced in the collision of a beam of $\mathrm{N}_{2}$ or $\mathrm{O}_{2}$ with a perpendicular beam of metal atoms. The definitive relationship for these cross sections is $\Phi_{\lambda}=F\left(1-e^{-N x \sigma}\right)$, where $\Phi_{\lambda}$ is the number of photons per second emitted at a wavelength $\lambda$ when a gas beam of intensity $F$ passes a distance $x$ through a metal beam of density $N$. A similar expression relates $\sigma_{i}$ to the number of ions produced.

\section{APPARATUS}

The crossed molecular beam apparatus used to make these measurements shown in figure 1 has been described in detail in our previous reports (Boitnott and Savage, 1970; Savage and Boitnott, 1971). Bricfly nitrogen or oxygen is ionized in the source and focused through the analyzing magnet to select the desired ion. The ion energy is set by

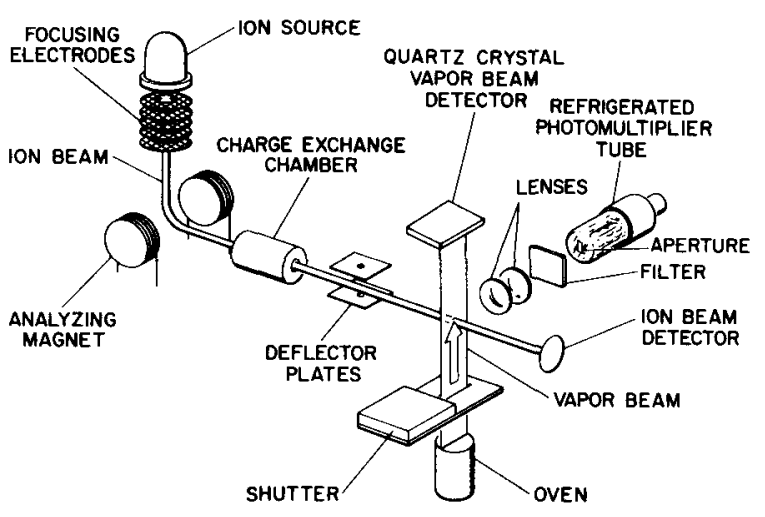

Frgure 1.-Schematic of emission cross-section apparatus.

the potential at the ion source. The resulting $\mathrm{N}_{2}^{+}$ or $\mathrm{O}_{2}+$ beam is then focused into a charge exchange cell which contains enough $\mathrm{N}_{2}$ or $\mathrm{O}_{2}$ to resonantly charge exchange about half of the original ion beam. In such a charge exchange process, there is no energy exchange or scattering as long as the densities are low enough so that there are no secondary collisions. The electrostatic deflection plates remove the remaining ions from the beam leaving only $\mathrm{N}_{2}$ or $\mathrm{O}_{2}$ to be injected into the test chamber. Intersecting at right angles to the $\mathrm{N}_{2}$ or $\mathrm{O}_{2}$ beam is the metal atom beam which emanates from a slit in an oven (the flux of metal atoms being determined by the oven temperature). The measurements were generally made with metal atom densities of the order of $10^{10} \mathrm{~cm}^{-3}$ while the background density of gas in the test chamber was about $5 \times 10^{10} \mathrm{~cm}^{-3}$. For $\mathrm{Na}$, the flux was measured with a hot tungsten oxide surface ionizer as described in our $\mathrm{Na}$ paper (Boitnott and Savage, 1970); and the $\mathrm{Mg}$, $\mathrm{Ca}$, and $\mathrm{Fe}$ fluxes were measured with an oscillating quartz crystal.

A portion of the photons generated by the collisions pass through a bandpass filter and are counted. The bandpass of these filters is typically $100 \AA$ and is centered on the multiplet of interest. Now, with the photon count, calibration factors, metal atom density, and gas beam intensity, the emission cross section is determined. Then with the ionization cross section determined from the ion count, we can work through the equations previously discussed and determine the luminous efficiency for the spectral feature being observed.

The conditions of the experiment which can be controlled are: 
(1) The densities of the colliding beams

(2) The energy involved in the collision

(3) The collision partners

(4) The photon wavelength observed.

In regard to the densities of the beams, all of our measurements have been made under free molecular flow conditions. As for the energy, unfortunately, our lowest energy is limited to about $150 \mathrm{eV}$ so that the luminous efficiency calculations must be made with extrapolation of the pertinent cross sections. Therefore, the efficiency values below about $30 \mathrm{~km} / \mathrm{s}$ are much more uncertain than at the higher velocities.

\section{EMISSION CROSS SECTIONS}

The emission cross sections of the most prominent multiplets of $\mathrm{Na}, \mathrm{Mg}$, and $\mathrm{Ca}$ are shown in figure 2 over the range of energies investigated. The wavelengths of the lines of each multiplet shown in figures 2,3 , and 4 are listed in table 1 . The data shown for $\mathrm{Fe}$ are for the total radiation in the wavelength range between 3500 and $4200 \AA$. The number of lines in the Fe spectrum is so numerous and closely spaced that bandpass filters could only make measurements for collections of multiplets and could not easily isolate individual multiplets. At wavelengths greater than $4200 \AA$, the blackbody radiation from the

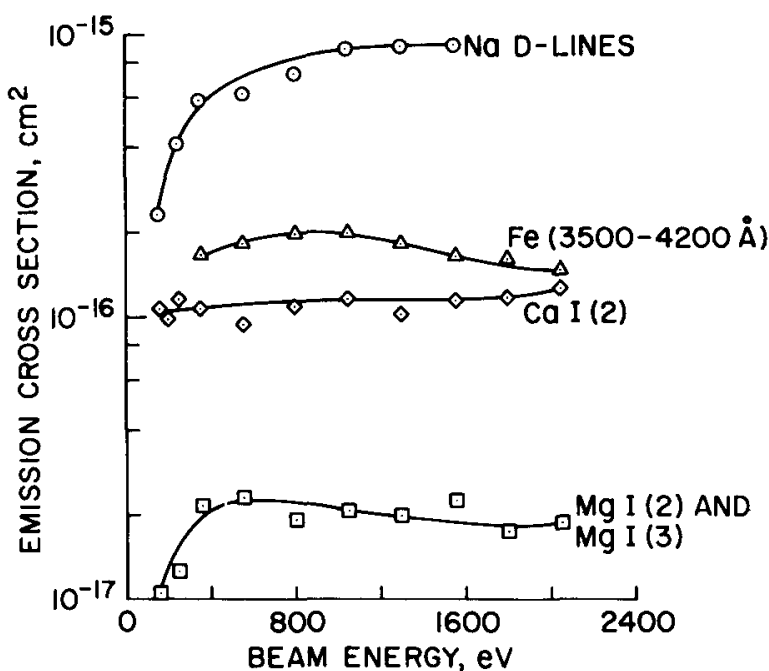

Figure 2.--Emission cross sections due to $\mathrm{N}_{2}$ collisions for the prominent multiplets of $\mathrm{Na}, \mathrm{Mg}$, and $\mathrm{Ca}$ and of Fe over the range 3500 to $4200 \AA$. source oven made meaningful measurements very difficult.

Cross sections for $\mathrm{Mg}$ and $\mathrm{Ca}$ shown in figure 2 are typical with other multiplets of these elements

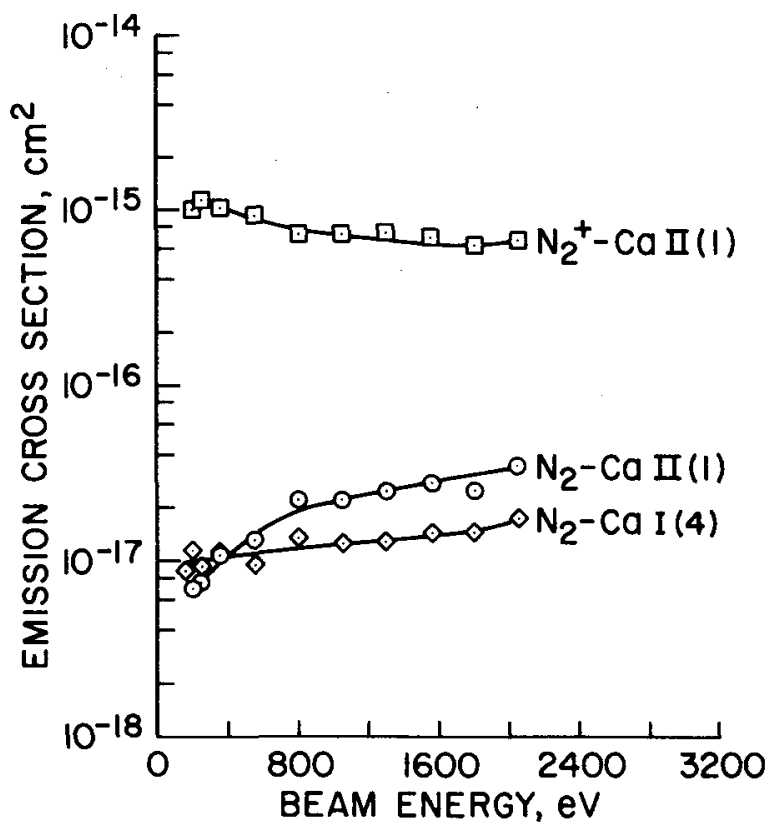

Figure 3.-Fmission cross sections of $\mathrm{Ca}$ due to $\mathrm{N}_{2}$ and $\mathrm{N}_{2}+$ collisions.

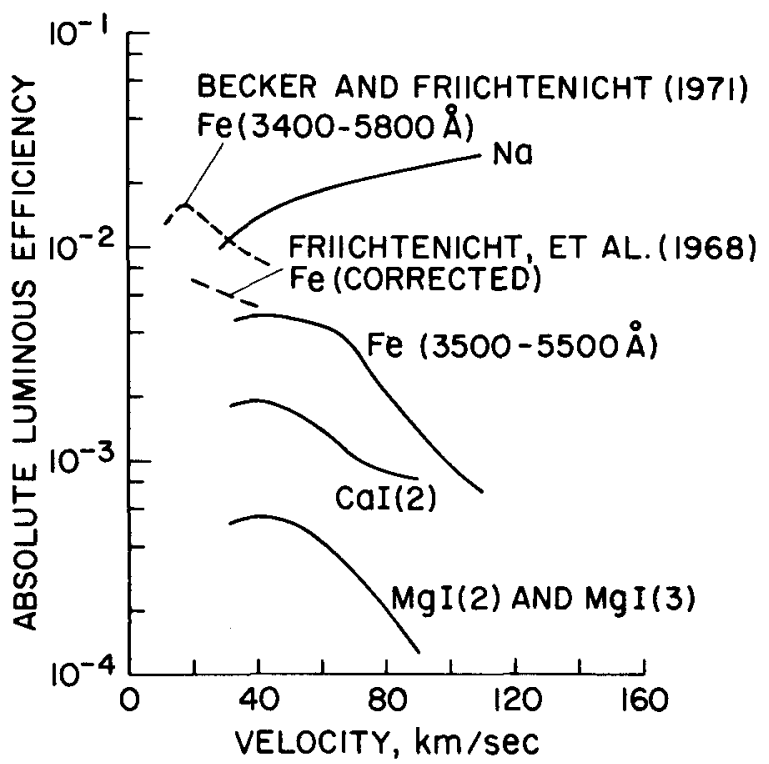

Figure 4.-Absolute luminous efficiencies for the prominent multiplets of $\mathrm{Na}, \mathrm{Mg}$, and $\mathrm{Ca}$ and of the visible spectrum of Fe. 
TABLE 1.-Wavelengths of Multiplet Lines

\begin{tabular}{ll}
\hline Multiplet & Line wavelengths, $\AA$ \\
\hline Na $D$-lines & 5890,5896 \\
CaI $(2)$ & 4227 \\
CaI $(4)$ & $4425,4435,4455$ \\
CaII(1) & $3934(K), 3938(H)$ \\
MgI(2) & $5167,5173,5183$ \\
MgI(3) & $3829,3832,3838$ \\
\hline
\end{tabular}

showing similar characteristics but with smaller magnitudes. Collisions involving $\mathrm{O}_{2}$ instead of $\mathrm{N}_{2}$ generally had lower cross sections, by as much as a factor of 5, and did not necessarily show the same variations with energy (Savage and Boitnott, 1971; Boitnott and Savage, 1970).

Of considerable interest are the $\mathrm{CaII}(1)$ lines, $\mathrm{H}$ and $\mathrm{K}$, since they frequently appear enhanced in meteor spectra and are thought to indicate slip flow conditions. The emission cross sections are shown in figure 3 as a function of energy for both $\mathrm{N}_{2}$ and $\mathrm{N}_{2}{ }^{+}$in collisions with neutral Ca. In addition to the large difference in magnitude between the ion and neutral collision partners, the variation with energy is completely different. The high $\mathrm{H}$ and $\mathrm{K}$ emission for $\mathrm{N}_{2}{ }^{+}$collisions with neutral $\mathrm{Ca}$ is due to near resonant charge exchange including excitation of the $\mathrm{Ca}$ ion in a single collision. Without getting into a complete discussion of the $\mathrm{H}$ and $\mathrm{K}$ line enhancement as the meteor gets lower in the atmosphere, we want only to show that even in the free molecular flow regime there is considerable $\mathrm{H}$ and $\mathrm{K}$ emission as a result of neutral-neutral collisions. For the luminous efficiency and a more complete discussion of the $\mathrm{H}$ and $\mathrm{K}$ enhancement, see Boitnott and Savage (1971). The cross sections indicate, that in the free molecular flow regime, the multiplets CaI(4) and CaII(1) should be of nearly equal intensity. Any enhancement of the $\mathrm{H}$ and $K$ lines should take place as the meteor enters the denser atmosphere where there are secondary collisions between neutral calcium and metal or gas ions, i.e., slip flow conditions.

\section{LUMINOUS EFFICIENCIES}

The absolute luminous efficiencies for the most prominent multiplets of $\mathrm{Na}, \mathrm{Mg}$, and $\mathrm{Ca}$ are shown in figure 4 along with the total luminous efficiency for the visible spectra of iron. For the luminous efficiencies of the other multiplets of $\mathrm{Mg}$ and $\mathrm{Ca}$, see Boitnott and Savage (1971). To obtain the total for iron, allowances were made for the visible spectrum above $4200 \AA$ by comparing our bandpass data in the 3500 to $4200 \AA$ range with the spectrum obtained by Tagliaferri and Slattery (1969) and with the natural meteor data of Harvey (1970). The decrease in the efficiencies of $\mathrm{Fe}, \mathrm{Mg}$, and $\mathrm{Ca}$ at the higher velocities is caused by the high relative probability of ionization at these velocities. This has the effect of removing neutral atoms which might be excited during a later collision in the deceleration process. Also the emission cross sections for the neutral atom lines due to collision between metal ions and air molecules are very low in comparison to those for neutral-neutral collisions. There is generally good agreement between our data and the free molecular flow meteor simulation experiments of Friichtenicht et al. (1968) and Becker and Friichtenicht (1971). The data of Friichtenicht et al. has been increased by 20 percent to account for the more accurate spectral distribution found later by Tagliaferri and Slattery (1969).

The luminous efficiencies we have determined for the multiplets of $\mathrm{Na}, \mathrm{Mg}$, and $\mathrm{Ca}$ and the

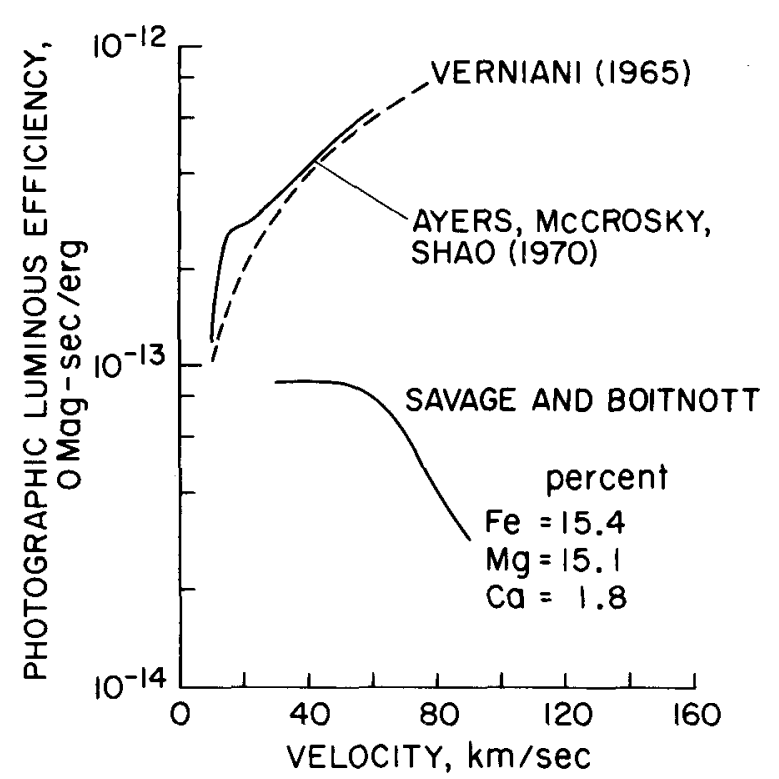

FIgURE 5.-The photographic luminous efficiency of a stone for blue sensitive film. 
total for iron are directly applicable to the determination of the relative abundances of these elements in those free molecular flow photographic meteors for which good resolution spectra are available. With an assumption on the relative abundances of the weak and nonradiating elements which make up the rest of the meteor, the mass can be determined.

As an exercise, we have calculated the photographic luminous efficiency of blue sensitive film (Ayers, 1965) for a meteor with the assumed composition of meteoric stone (fig. 5). Variations in the composition would have only minor effects on the magnitude of $\tau$ without changing the general trends. Other vaporized elements present in the meteor are assumed to provide negligibly weak radiation and are neglected. In addition, any contribution by air molecules is not included in the calculations. Such radiation is toward the red and is seen in spectra using panchromatic film as are the $\mathrm{Na} D$-lines.
For comparison, we show the luminous efficiency deduced by Verniani (1965) from 413 photographic meteors where he assumed that $\tau$ is proportional to the $V^{n}$, for all velocities, and concluded that $n=1$. Also shown is the best guess efficiency of Ayers et al. (1970) which is primarily based on low velocity, near continuum flow, artificial meteor data. The reasons for the large differences are not clear to us at this time.

In summary, we have measured in the laboratory the fundamental emission and ionization cross sections needed to determine the spectral luminous efficiencies of $\mathrm{Na}, \mathrm{Mg}$, and $\mathrm{Ca}$ and the total efficiency for iron. We are encouraged by the good agreement with the luminous efficiency of iron measured by Friichtenicht et al., and Becker and Friichtenicht, and feel that the luminous efficiencies obtained can be applied to the quantitative analysis of meteor spectra in free molecular flow conditions.

\section{REFERENCES}

Ayers, W. G., 1965. Luminous efficiency of an artificial meteor at 11.9 kilometers per second, NASA TN D-2931.

Ayers, W. G., MCCrosky, R. E., and Shao, C. -Y., 1970. Photographic observations of 10 artificial meteors, Smithson. Astrophys. Obs. Spec. Rept. No. 317.

Becker, D. G., and Frichtenicht, J. F., 1971. Measurement and interpretation of the luminous efficiencies of iron and copper simulated micrometeors, Astrophys. J., 166, 699-716.

Boitnot, C. A., ANd SAvage, H. F., 1970. Light-emission measurements of sodium at simulated meteor conditions, Astrophys. J., 161, 351-358.

Boitnott, C. A., ANd Savage, H. F., 1971. Light-emission measurements of calcium and magnesium at simulated meteor conditions. II. Spectral luminous efficiencies, Astrophys. J., 167, 349-355.

Frimchtenicht, J. F., Slattery, J. C., and Tagliafferri, E., 1968. A laboratory measurement of meteor luminous efficiency, Astrophys. J., 151, 747-758.

HaRvey, G. A., 1970. Spectra of faint optical meteors, unpublished.

Savage, H. F., And Bortnotr, C. A., 1971. Light-emission measurements of calcium and magnesium at simulated meteor conditions. I. Cross-section measurements, Astrophys. J., 167, 341-348.

SidA, D. W., 1969. The production of ions and electrons by meteoritic processes, Monthly Notices Roy. Astron. Soc., 155, 37-47.

Tagliaferri, E., AND Slattery, J. C., 1969. A spectral measurement of simulated meteors, Astrophys. J., 155, 1123-1127.

Verniani, F., 1965. On the luminous efficiency of meteors, Smithson. Contrib. Astrophys., 8, 141-172. 